\title{
Density of Localized States near the Band Edge of Disordered Systems
}

\author{
Zhao-Qing Zhang ${ }^{(a)}$ and Ping Sheng \\ Corporate Research Science Laboratories, Exxon Research \& Engineering Company, Annandale, New Jersey 08801
}

(Received 14 May 1986)

\begin{abstract}
The band-tail density of states for the tight-binding model with Gaussian distribution of site energies is calculated numerically in one, two, and three dimensions by use of the recursive transformation method. Our results confirm the band-tail scaling properties as predicted by the HalperinLax theory but indicate that in $3 \mathrm{D}$, the near-tail behavior displayed by the model is qualitatively distinct from the simple exponential as deduced from experimental data. Implications are discussed.
\end{abstract}

PACS numbers: $71.20 .+\mathrm{c}, 71.50 .+\mathrm{t}$

Study of band tails in disordered systems has been a topic of continuing experimental and theoretical interest over the past three decades. From optical and electrical measurements on a wide variety of disordered semiconductors, ${ }^{1,2}$ it has been deduced that the density of states $\rho(E)$ in the tail region can be described by the functional form $\ln \rho(E) \sim\left(E-E_{1}\right)^{\alpha}$, where $E_{1}$ is a constant in the vicinity of the band edge, and $\alpha=1$. Theoretical modeling of the band-tail behavior, on the other hand, is far less conclusive as a result of the lack of exact results even for simple models. In their classical work, Halperin and Lax $^{3}$ (HL) have presented a variational approach to the calculation of band-tail densities of states. In one dimension, the predictions of the HL theory have been corroborated by more exact treatment ${ }^{4,5}$ of the problem. However, in three dimensions a discrepancy emerges between the HL density-of-states behavior, $\ln \rho(E)$ $\sim\left(E-E_{1}\right)^{1 / 2}$, and that deduced from experimental data. A number of suggestions have been made recently in regard to the source of this discrepancy. ${ }^{6-8}$ While the ideas vary, the implicit basic position remains that the exponential tail of $\rho(E)(\alpha=1)$ should be explainable solely on the basis of random potential fluctuations. Therefore the general overriding concern is to obtain the true density-of-states behavior for the $3 \mathrm{D}$ random potential model. In this work we use the recursion method devoped by Haydock, Heine, and Kelly ${ }^{9}$ to calculate directly the tail density of states in $d=1,2$, and 3 for a tight-binding Hamiltonian with Gaussian distribution of site energies. Our results confirm the band-tail scaling properties predicted by the HL theory as well as delineate the regime of applicability for the coherent potential approximation to the tail region. However, in 3D the calculated $\ln \rho$ is shown to display characteristics which are qualitatively distinct from the experimentally deduced $\alpha=1$ behavior. This disagreement suggests that either there is some aspect of the tail density-of-states behavior which is not yet experimentally observed, or else some elements in addition to random potential fluctuations must be taken into consideration for a proper account of the tail behavior.

$$
\begin{aligned}
& \text { Consider the tight-binding Hamiltonian } \\
& \qquad H=\sum_{i} \epsilon_{i}|i\rangle\left\langle i\left|+V \sum_{[i, j]}\right| i\right\rangle\langle j|,
\end{aligned}
$$

where the constant hopping matrix element $V$ will be set equal to $1,[i, j]$ denotes the nearest-neighbor pair, and $\epsilon_{i}$ is the random site energy which is Gaussian distributed with mean zero and variance $w^{2}$. The use of Gaussian distribution for $\epsilon_{i}$ is based on the reasonable assumption, supported by recent experimental evidence, ${ }^{10}$ that fluctuations in $\epsilon_{i}$ arise from independent additive noise sources. The electronic density of states of the system is formally given by

$$
\rho(E)=-\frac{1}{\pi} \lim _{\eta \rightarrow 0^{+}} \operatorname{Im}\left\langle G_{0}^{(\gamma)}(E+i \eta)\right\rangle_{\gamma},
$$

where $\gamma$ is a configuration index, \langle\rangle$_{\gamma}$ means configurational averaging, and $G_{0}^{(\gamma)}$ is the Green's function determined at the origin (the source point) for configuration $\gamma$. To calculate $G_{0}^{(\gamma)}(E+i \eta)$, we use the recursion method developed by Haydock, Heine, and Kelly ${ }^{9}$ to transform the equation of motion into a tridiagonal form in the new basis representation. The new equation of motion is truncated at the $N$ th basis function by the introduction of a self-energy $\Sigma^{(\gamma)}(E)$, and the true Green's function $G_{0}^{(\gamma)}(E+i \eta)$ is approximated by $G_{0}^{(\gamma)}\left(E+i \eta, \Sigma^{(\gamma)}(E), N\right)$. The self-energy $\Sigma^{(\gamma)}(E)$ is determined through the self-consistent condition

$$
\begin{aligned}
G_{0}^{(\gamma)}\left(E+i \eta, \Sigma^{(\gamma)}\right. & (E), N) \\
& =G_{0}^{(\gamma)}\left(E+i \eta, \Sigma^{(\gamma)}(E), N-1\right) .
\end{aligned}
$$

From $G_{0}^{(\gamma)}\left(E+i \eta, \Sigma^{(\gamma)}(E), N\right)$ we can obtain the local density of states as a function of $E$. An averaging over configurations then yields $\rho(E)$. In actual implementation both the number of basis functions $N$ and the number of configurations considered in the averaging process were systematically increased till the value of the tail density of states stabilized. In all calculations a small $\eta$, on the order of $10^{-5}$ to $10^{-4}$, was added to $E$ so that states which appear as isolated poles in $G_{0}^{(\gamma)}(E, \Sigma, N)$ may be picked up. Also, since the 
$\rho(E)$ for the tight-binding model should be symmetrical about $E=0$, the density-of-states statistics can be improved by an averaging of $G^{(\gamma)}(E, \Sigma, N)$ with $G_{0}^{(\gamma)}(-E, \Sigma, N)$ and use of the resulting Green's function in Eq. (2).

In one dimension, our direct calculation of $\rho(E)$ can be compared with the perturbation solution ${ }^{5}$ of the exact density-of-states equation, i.e.,

$$
\begin{aligned}
& \bar{\rho}(\bar{E})=(2 / \pi)^{1 / 2} N_{+} / N_{-}^{2}, \\
& N_{ \pm}=\int_{0}^{\infty} d t t^{ \pm 1 / 2} \exp \left(-\frac{1}{6} t^{3}+2 \bar{E} t\right),
\end{aligned}
$$

where $\bar{\rho}=\rho w^{2 / 3} v^{1 / 3} a$ is the scaled dimensionless density of states, $a$ is the lattice constant (set equal to 1 ), and $\bar{E}=(E-2) /\left(w^{4 / 3} v^{-1 / 3}\right)$ is the scaled dimensionless energy with the crystalline band edge $(E=2)$ as the reference origin. Figure 1 shows a plot of $\bar{\rho}(\bar{E})$, Eq. (3), and the results of numerical calculation carried out for $w=0.6, N=40$ (81 sites), averaged over $4.3 \times 10^{6}$ configurations. The agreement is excellent for $\bar{E}<1.5$, but the perturbation solution seems to underestimate slightly the density of states deeper in the tail region. It should be noted that Eq. (3) yields exactly the HL density of states, $\rho_{\mathrm{HL}}(\bar{E})=(4 \bar{E} / \pi)$ $\times \exp \left(-8 \bar{E}^{3 / 2} / 3\right)$, in the asymptotic limit of large $\bar{E}$.

In two dimensions, there is no exact theory for the $\rho(E)$ tail behavior. Our results will be compared with the coherent potential approximation ${ }^{11}$ (CPA) and the HL theory. For the CPA,

$$
\begin{aligned}
& \rho_{\mathrm{CPA}}(E)=-\pi^{-1} \operatorname{Im} G_{c}\left(E-\Sigma_{\mathrm{CPA}}\right), \\
& \Sigma_{\mathrm{CPA}}(E)=\int_{-\infty}^{\infty} d \epsilon_{i} \frac{P\left(\epsilon_{i}\right) \epsilon_{i}}{1-\left(\epsilon_{i}-\Sigma_{\mathrm{CPA}}\right) G_{c}\left(E-\Sigma_{\mathrm{CPA}}\right)},
\end{aligned}
$$

where $G_{c}$ denotes the crystalline Green's function for the square lattice in our present application, and $P\left(\epsilon_{i}\right)$

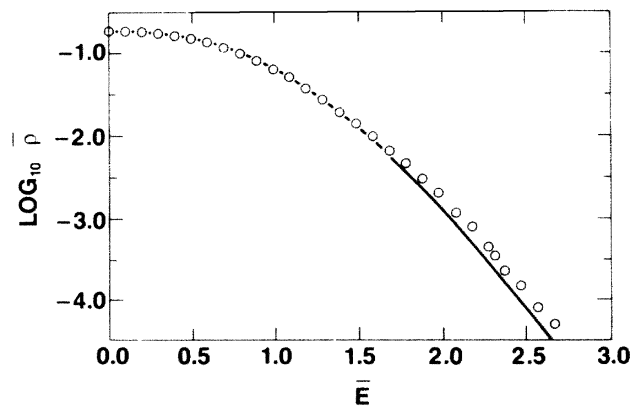

FIG. 1. Plot of scaled 1D tail density of states as a function of scaled energy. Open circles denote numerical results calculated with $w=0.6, N=40$ (81 sites), and averaged over $4.3 \times 10^{6}$ configurations. Solid line is calculated from Eq. (3). The maximum error bar on our numerical results is smaller than the size of the open circle. is the Gaussian distribution of $\epsilon_{i}$. For the HL theory, ${ }^{12}$ we have

$$
\bar{\rho}_{\mathrm{HL}}(\bar{E})=0.120 \bar{E}^{0.5689} \exp (-0.9311 \bar{E}),
$$

where $\bar{\rho}=\rho 4 \pi V a^{2}, \bar{E}=\left(E-E_{\mathrm{CPA}}\right) 4 \pi V / w^{2}$, and $E_{\mathrm{CPA}}$ is the band edge determined from the truncated CPA equation $^{13}$

$$
\Sigma=w^{2} G_{c}(E-\Sigma)+w^{4} G_{c}^{3}(E-\Sigma) .
$$

In Fig. 2 the scaled density of states $\bar{\rho}$, calculated on the simple square lattice, is plotted as a function of scaled energy $\bar{E}$ for five different values of $w=0.2$, $0.4,0.6,0.8$, and 1.0. For two of the values, $w=0.2$ and 0.6 , we have also plotted the density of states calculated on finite clusters (embedded in vacuum). The purpose of the finite-cluster calculations is to check effect of different boundary conditions on our results. It is found that as long as both the cluster size $(N>30)$ and the number of clusters averaged are sufficiently large, the results obtained are insensitive to the boundary conditions. The fact that in the averaging process only the Green's function at the origin (center of the cluster) is involved also diminishes

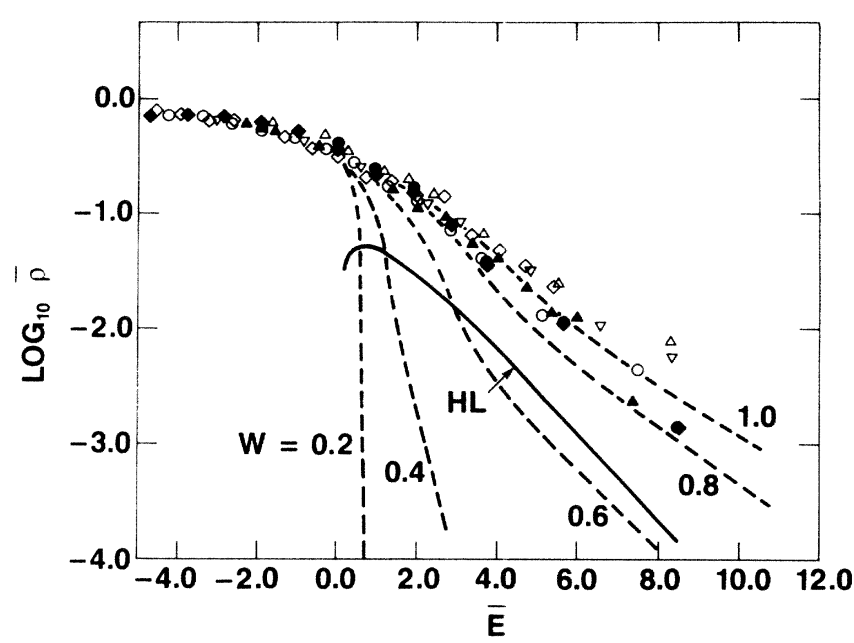

FIG. 2. Scaled 2D tail density of states plotted as a function of scaled energy. Solid lozenges, $w=0.2, N=35$ (2521 sites), $10^{3}$ configurations; open circles, $w=0.4, \quad N=31$ (1985 sites), $1.2 \times 10^{4}$ configurations; open lozenges, $w=0.6, N=31$ (1985 sites), $4 \times 10^{3}$ configurations; inverted triangles, $w=0.8, N=31$ (1985 sites), $2 \times 10^{4}$ configurations; solid triangles, $w=1.0, N=31$ (1985 sites), $2 \times 10^{4}$ configurations; solid circles, finite cluster $w=0.2, N=39$ (3121 sites), $6.6 \times 10^{3}$ configurations; open triangles, finite cluster $w=0.6, N=39$ (3121 sites), $2 \times 10^{4}$ configurations. The solid line is the HL theory prediction calculated from Eq. (5). The dashed lines are the CPA results evaluated from Eq. (4) with different values of $w$. The error bar on our numerical results varies from less than the size of the symbol for $\bar{E}<0$ to about twice the size of the symbol at $\bar{E} \simeq 8$. 
the influence of the boundary.

We note in Fig. 2 that the scaling of the different $w$ curves implied by Eq. (5) [ $\bar{\rho}$ is only a function of $\left.\left(E-E_{\mathrm{CPA}}\right) / w^{2}\right]$ is well followed by the numerical data in the range of $\bar{E}<5$. While the prediction of Eq. (5) falls below the data in terms of magnitude, the slope of the solid line reasonably parallels that of the calculated data points. Only at large $w$ values is there a systemtic deviation of the data from the HL slope. In this context it should be mentioned that the numerical density of states results of Thouless and Elzain, ${ }^{12} \mathrm{ob}-$ tained from finite clusters of up to 7100 sites (with an equivalent $w$ value of 0.58 ), are in the range of $\bar{E}<3$ and have magnitudes which fall consistently below our calculated values. The difference is attributable to the fact that a square distribution of $\epsilon_{i}$ was used in their numerical work. An independent calculation by us has verified this effect due to the shape of the $\epsilon_{i}$ distribution.

Comparison between our 2D results and the CPA curves as evaluated from Eq. (4) is seen to show remarkable agreement for $\bar{E}<0$, i.e., within the CPA band. In the tail region the CPA results display no scaling behavior, and the agreement with our numerical results is poor for $w<0.8$. However, as $w$ increases beyond 0.8 the agreement improves. A plausible explanation for this fact is that as $w$ increases, more and more states tend to be localized on a single (or a few) site(s), and CPA's single-site effective medium description thereby becomes more accurate. Since the HL theory inherently treats the other limit where each localized state extends over many sites, it is interesting to note from Fig. 2 that in $2 \mathrm{D}, w=0.6$ seems to be the dividing value above which the tightly localized states dominate the density-of-states behav-

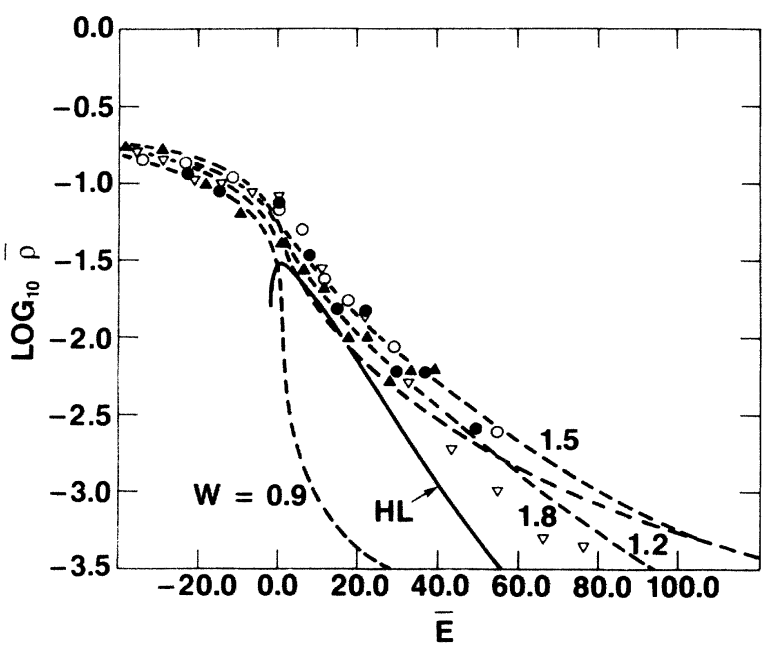

FIG. 3. Scaled 3D tail density of states plotted as a function of scaled energy. Open circles, $w=0.9,2.5 \times 10^{3}$ configurations; inverted triangles, $w=1.2,4 \times 10^{3}$ configurations; solid circles, $w=1.5,10^{4}$ configurations; solid triangles, $w=1.8,1.4 \times 10^{4}$ configurations. In all the numerical calculations we used $N=29$ (34279 sites). The solid line is the HL theory prediction calculated from Eq. (6). The dashed lines denote the CPA results evaluated from Eq. (4) with the values of $w$ given in the figure. The error bar on our numerical results varies from less than the size of the symbol for $\bar{E}<0$ to about twice the size of the symbol at $\bar{E} \simeq 70$.

ior in the near-tail region, and below which the largersized localized states are more numerous.

In Fig. 3 we present four sets of 3D tail density of states calculated on a simple cubic lattice with $w=0.9$, $1.2,1.5$, and 1.8. For comparison, predictions of the HL theory and the CPA are also plotted. The HL density of states has the form ${ }^{13,14}$

$$
\bar{\rho}_{\mathrm{HL}}(\bar{E})=\bar{\rho}_{\mathrm{CPA}}(8.96 / \pi+\bar{E}) 3.092(8.96 / \pi+\bar{E}) \exp \left[-1.504(8.96 / \pi+\bar{E})^{1 / 2}\right],
$$

where $\bar{\rho}=\rho 8 \pi v^{3} a^{3} / w^{2}, \quad \bar{E}=64 \pi^{2} v^{3}\left(E-E_{\mathrm{CPA}}\right) / w^{4}$, and the factor $8.96 / \pi$ gives the separation of the mobility edge from the CPA band edge. The CPA density of states is given by the same Eq. (4) except now $G_{c}$ stands for the crystalline Green's function in the simple cubic lattice. Again, we see that the scaling of the different $w$ curves predicted by Eq. (6) $[\bar{\rho}$ is a function only of $\left.\left(E-E_{\mathrm{CPA}}\right) / w^{4}\right]$ is borne out by the data, although in magnitude $\bar{\rho}_{\mathrm{HL}}$ tends to underestimate the numerical results. The behavior of the CPA band tails is similar to that seen in 2D: Agreement with the data improves as $w$ increases beyond 1 . The crisscrossing of the various CPA curves is also understandable since the slope of the CPA tail scales as $w^{2}$, whereas the near-tail behavior scales as $w^{4}$.

An important point to be noted about Fig. 3 is that the numerically calculated $\ln \bar{\rho}(\bar{E})$ distinctly displays an inflection point in the vicinity of $\bar{E}=0$. That is, $\ln \bar{\rho}$ changes from a downward curvature for $\bar{E}<0$ to an upward curvature at some point $\bar{E}>0$. In fact, a least-squares fit of our results with the form $\ln \bar{\rho}=a\left(E-E_{\mathrm{CPA}}\right)^{\alpha}+b$ yields exponent values of $\alpha=0.5,0.6,0.8$, and 0.5 for $w=0.9,1.2,1.5$, and 1.8 , respectively. These results clearly point out that the existence of an inflection point in $\ln \rho$ vs $E$ plots is a general qualitative feature of the density of states for the 3D random-potential model and should therefore be experimentally observable if potential fluctuations are the only underlying physics of the phenomena. In this regard we note that our results support the qualitative near-tail features of the HL theory and the CPA, since in the region of $\bar{E} \geq 0$ both show positive curvatures for $\ln \bar{\rho}$. For CPA, however, the point of inflec- 
tion can become less and less obvious and eventually disappear as $w$ increases beyond 2 .

Recently the works of Abe and Toyozawa, ${ }^{15}$ Economou et al., ${ }^{13}$ Sritrakool, Sa-yakanit, and Glyde, ${ }^{7}$ and John et al. ${ }^{8}$ have all shown that in the deep-tail region, i.e., $\bar{E}>40$, both the CPA and a continuum model of potential fluctuations with finite correlation length can yield $\alpha=1$ behavior over an extended energy range. Comparison with experimental results also yields reasonable values for the parameters of the theories. Since in the limit of $\bar{E} \rightarrow \infty$ both the CPA and the field theoretical solution of the continuum model are asymptotically exact, ${ }^{8,13}$ their extension to values of $\bar{E}$ in the deep-tail region should be reasonably accurate. This implies that together with our numerical simulation results in the near-tail region, there is now a complete picture of tail density of states for the $3 \mathrm{D}$ random-potential model over the entire energy range. In order of increasing $E$, the three essential features of this picture consist of (1) an inflection point $(\alpha<1)$ in the near-tail region, (2) an extended tail region with $\alpha=1$, and (3) the eventual Gaussian decay with $\alpha=2$. If we assume that the $\alpha=2$ segment would be masked by other processes, then in order for the above picture to account for the experimental results it is crucial to ask whether the $\alpha<1$ near-tail behavior and its implied inflection point have been experimentally observed in the energy range where the band tail joins onto the band edge. To our knowledge, there is yet no experimental support in the literature for this type of near-tail behavior. ${ }^{1,2}$ Therefore, besides urging the reexamination of experimental data in light of the new evidence presented in this work, we would also like to speculate that if the qualitative behavior of $\alpha<1$ is not observed or is observed only in a limited class of materials, then there could be mechanisms in addition to random potential fluctuations which play a significant role in the near-tail region. Since the random-potential model neglects electron-electron and (the dynamical aspect of) electron-phonon interactions, the effects of these interactions on the tail density of states and the optical absorption edge suggest themselves as a natural direction for further investigation. In fact, a combination of these interactions has been proposed as the mechanism responsible for the phenomenon of Urbach tail. ${ }^{16}$
In conclusion, we have performed direct numerical calculation of the band-tail density of states for the tight-binding, random potential model in $d=1,2$, and 3. Besides confirming the scaling behavior of $\rho(E)$ and providing a basis of comparison for the various phenomenological theories, our results suggest that the intrinsic near-tail behavior of the $3 \mathrm{D}$ random potential model is distinct from that deduced from experimental data, thereby implying a more complex origin for the exponential tail.

The authors wish to acknowledge helpful discussions with E. N. Economou, M. Cohen, C. Soukoulis, G. Cody, and S. John.

(a) On leave from Department of Physics, San Francisco State University, San Francisco, CA 94132, and Institute of Physics, Academia Sinica, Beijing, People's Republic of China.

1J. D. Dow, Comments Solid State Phys. 4, 35 (1972).

${ }^{2}$ G. D. Cody, Semiconductors and Semimetals (Academic, New York, 1985), Vol. 21, Pt. B, p. 11.

${ }^{3}$ B. I. Halperin and M. Lax, Phys. Rev. 148, 722 (1966).

${ }^{4}$ B. I. Halperin, Phys. Rev. 139, A104 (1965).

${ }^{5}$ B. Derrida and E. Gardner, J. Phys. (Paris) 45, 1223 (1984).

${ }^{6}$ C. Soukoulis, M. Cohen, and E. N. Economou, Phys. Rev. Lett. 53, 616 (1984).

7W. Sritrakool, V. Sa-yakanit, and H. R. Glyde, Phys. Rev. B 33, 1199 (1986).

${ }^{8}$ S. John, C. Soukoulis, M. Cohen, and E. N. Economou, to be published.

${ }^{9}$ R. Haydock, V. Heine, and J. J. Kelly, J. Phys. C 5, 2845 (1972).

${ }^{10}$ G. D. Cody, T. Tiedje, B. Abeles, B. Brooks, and Y. Golstein, Phys. Rev. Lett. 47, 1480 (1981).

${ }^{11}$ For a description of CPA and its applications, see E. N. Economou, Green's Functions in Quantum Physics (SpringerVerlag, New York, 1983), 2nd ed., Chap. 7.

${ }^{12}$ The slope of the exponential decay in Eq. (5) was first calculated by D. J. Thouless and M. E. Elzain, J. Phys. C 11, 3425 (1978). The preexponential factor was obtained by E. Brézin and G. Parisi, J. Phys. C 13, L307 (1980).

${ }^{13}$ E. N. Economou, C. M. Soukoulis, M. H. Cohen, and A. D. Zdetsis, Phys. Rev. B 31, 6172 (1985).

${ }^{14}$ E. Brézin and G. Parisi, in Ref. 11.

${ }^{15} \mathrm{~S}$. Abe and Y. Toyozawa, J. Phys. Soc. Jpn. 50, 2185 (1981)

16D. Redfield and J. D. Dow, Phys. Rev. B 5, 594 (1972). 